\title{
Polydimethylsiloxane as a Potential Antenna Substrate
}

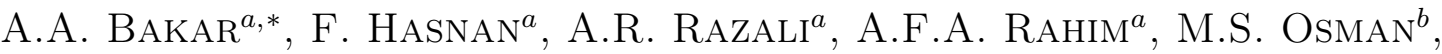 \\ T. ALI ${ }^{c}$ AND R. RADZALI ${ }^{a}$ \\ ${ }^{a}$ Faculty of Electrical Engineering, Universiti Teknologi Mara Pulau Pinang, \\ 13500 Permatang Pauh, Pulau Pinang, Malaysia \\ ${ }^{b}$ Faculty of Chemical Engineering, Universiti Teknologi Mara Pulau Pinang, \\ 13500 Permatang Pauh, Pulau Pinang, Malaysia \\ ${ }^{c}$ Faculty of Electrical Engineering, Universiti Teknologi Mara, 40450 Shah Alam, Malaysia
}

\begin{abstract}
The objective of this study is to investigate the potential material, PDMS as a flexible antenna substrate for body centric wireless application. A stretchable and flexible antenna design fabricated with thin copper as the radiating element and using the polydimethylsiloxane as the substrate is presented. Thin copper sheet based conductor has been a good material due to its low cost and high electrical conductivity. Polydimethylsiloxane is a flexible substrate are that are inexpensive and able to withstand mechanical strains. The measured dielectric constant of polydimethylsiloxane is about 2.76 to 3.00 across the measured frequencies. In the antenna design, the ground plane is separated from the transmission line and patch of the antenna by the dielectric substrate. Polydimethylsiloxane acts as an encasing the patch radiator and ground plane. Copper appeared as a radiator while polydimethylsiloxane as a substrate. The targeted resonant frequency of the designed antenna is $2.4 \mathrm{GHz}$ and has been achieved in the simulation using CST software. From the dielectric constant measurement, the relative permittivity $\varepsilon_{r}$ of polydimethylsiloxane was found to be 2.76 to 3.00 across the measured frequency and lossy tangent, $\tan \delta$ of 0.01 to 0.05 . The designed antenna has shown a good return loss performance and radiation pattern.
\end{abstract}

DOI: 10.12693/APhysPolA.135.938

PACS/topics: microstrip patch, polydymethylsiloxanes (PDMS), flexible antenna

\section{Introduction}

In recent years, there is an increasing need for new technologies such as flexible electronics. The demands of flexible antennas have received a huge attention use in a different kind of wearable applications. For modern application, wearable antenna needs a light weight construction, easily installation, low cost and practically maintenance free [1]. Stretch ability is the most demanding in a development of flexible antenna $[2,3]$.

Wearable antennas need a capability to be performed under various conditions while being worn. Conventional antennas are fabricated by printing or etching conductor patterns on rigid substrates, which lead fail to operate properly through bending, twisting and stretching process. Fabric-based antenna is fit use for body centric wireless communication (BCWC). It is also known to be more complex to environmental circumstance. Their performance will affect the operation under certain state.

Substrate contributes a larger part of an antenna which is lead to the flexibility of antenna [4]. Some of the substrate is polymer, fabric, paper and plastic. The polymer-based material becomes a most popular in flexible antenna development. Polymer-based antenna, which is combines highly conductive radiating element have

*corresponding author; e-mail: aslina060@uitm.edu.my been established well performance due to mechanical deformations and having a unique characteristic. Latest innovation in flexible antennas comprises thin film metal deposited on polydimethylsiloxane (PDMS) which is silicon-based elastomer with desirable characteristics such as flexibility, transparency and water resistance [5].

This trend can be widely explained by PDMS characteristics, which is chemically inert, thermally stable, permeable to gases, easy to handle and exhibits isotropic and homogenous properties [6]. PDMS also tremendous water isolator thus qualifying long-term, stable device process. PDMS has good RF and mechanical features, a low dielectric persistent and a good chemical strength. The preliminary liquid state of the PDMS permits preparation of composite substrates of several concentrations, thus adjusting the range of dielectric constants that can be acquired $[5,7]$. PDMS are inexpensive and capable to endure mechanical tensions when acts as a flexible substrate.

Through this study, new method had been proposed to design antennas using PDMS. To modify the dielectric permittivity of the substrate, the idea by adding inclusions to the PDMS has been developed [7]. By lowering the permittivity, the radiation characteristics can be improved [8]. Other than that, to manufacture a flexible and sealed antenna, integrate substrate and conductive parts had been proposed. To this aim, thin copper sheet will be used for the conductive parts, keeping the antenna flexibility. 


\section{Antenna design}

\subsection{Simulation using computer simulation technology}

Computer simulation technology (CST) studio provides a $3 \mathrm{D}$ electromagnetics simulation that deals with accurate, efficient computational solutions for electromagnetics design and analysis for this project.

To achieve the exact dimension, numerical analysis is crucial before undergo the antenna fabrication. The numerical analysis involved several stages: (i) creating the substrates layer (ii) creating the ground plane (iii) creating patch and running the antenna analysis. Once the dimensions have been confirmed as required according to the specification, the next step is to design the mould and fabrication process. Finally, the return loss of the fabricated antenna will be measured using vector network analysis (VNA) and the results will be compared with simulation results. Figure 1 shows the final antenna geometry after calculation and simulation are done. Table I shows the optimized dimensions of the designed antenna.

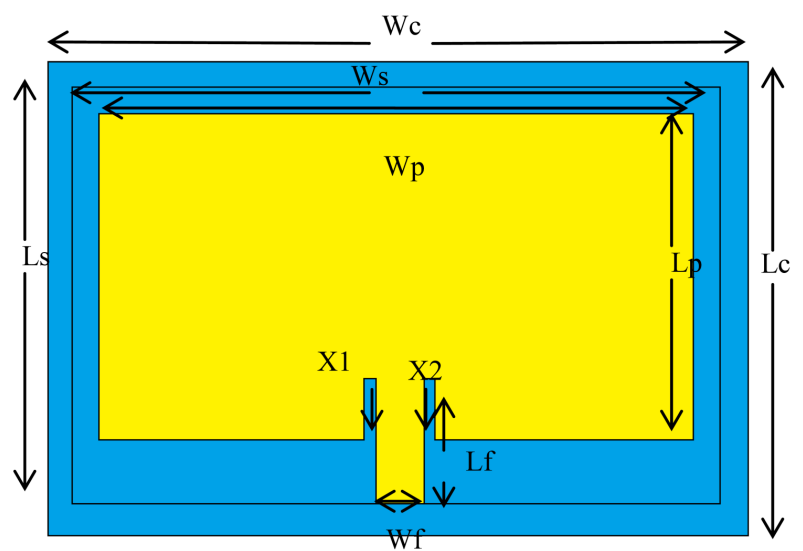

Fig. 1. Antenna geometry.

Antenna simulations parameters.

TABLE I

\begin{tabular}{l|c}
\hline \hline \multicolumn{1}{c|}{ Parameter } & Dimension [mm] \\
\hline patch length, $L_{p}$ & 35 \\
patch width, $W_{p}$ & 43 \\
patch height, $H_{p}$ & 0.5 \\
ground length, $L_{g}$ & 42 \\
ground width, $W_{g}$ & 52 \\
ground height, $H_{g}$ & 0.5 \\
substrates length, $L_{s}$ & 42 \\
substrates width, $W_{s}$ & 52 \\
substrates height, $H_{s}$ & 1.5 \\
inset feed length, $L_{f}$ & 8.17 \\
inset feed width, $X$ & 1 \\
feed width, $W_{f}$ & 3.6 \\
antenna cover length, $L_{c}$ & 44 \\
antenna cover width, $W_{c}$ & 54 \\
antenna cover height, $H_{c}$ & 3.2
\end{tabular}

\subsection{Substrates}

The targeted resonant frequency of the antenna is $2.45 \mathrm{GHz}$. Current study on PDMS dielectric characteristics stated that the relative permittivity $\varepsilon_{r}$ of 2.76 to 3.00 and lossy tangent $\tan \delta 0.01$ to 0.05 that operate at 0.2 to $5 \mathrm{GHz}$ [1]. The proposed antenna was designed with the relative permittivity $\varepsilon_{r}$ of 2.76 and lossy tangent $\tan \delta$ of 0.02 respectively.

Sylgard-184 PDMS was used for the substrate, where it will be in liquid states initially then get hardens after proper polymerized added. In order to get a good harden condition, the polymerization process will lasts one day by adding a curing agent. The dielectric constant of PDMS as stated above will gives a larger bandwidth and higher directivity but low in return loss as proved by L.C Paul et al. [7] as compared with other material with higher dielectric constant.

\subsection{Conductive material}

In this paper, copper $(\mathrm{Cu})$ plane is proposed as a conductive material due to it is low cost metal and high conductivity in microwave application. Copper act as a ground plane with the thickness of $0.5 \mathrm{~mm}$. Effectiveness of antenna matching by inset feed method can be achieved with $50 \Omega$ by varying the inset feed length. The ground plane is separated from the transmission line and patch of the antenna by the dielectric substrate. PDMS acts as an encasing the patch radiator and ground plane.

Figure 2 shows the cross section layers of the proposed antenna.

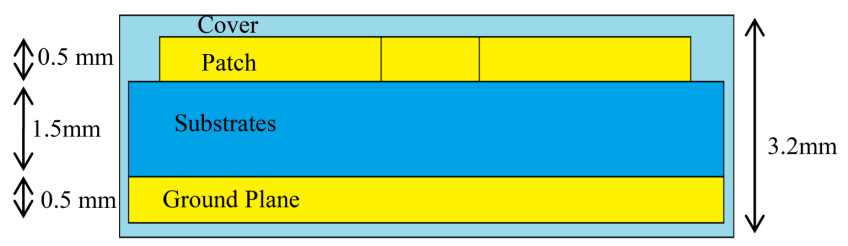

Fig. 2. Cross section layers.

\subsection{Mould preparation}

The technique used to design the mould is Rapid Prototyping where a scale model was fabricated or assembled using 3D Computer Aided Design (CAD). Through this process, a data gathering in terms of $x, y, z$ coordinates was transformed into numerous frame sets of high resolution 3D model. Data collections obtain by export the CST file format to standard triangulated language (STL) or computer aided design (CAD) file to be applied on Solidworks software.

Figure 3 shows the fabricated mould used in producing the antenna. In the first attempt in moulding, the layer of PDMS in both mould was distributed unevenly hence affect the thickness of the PDMS. The final fabricated antenna is shown in Fig. 4. 


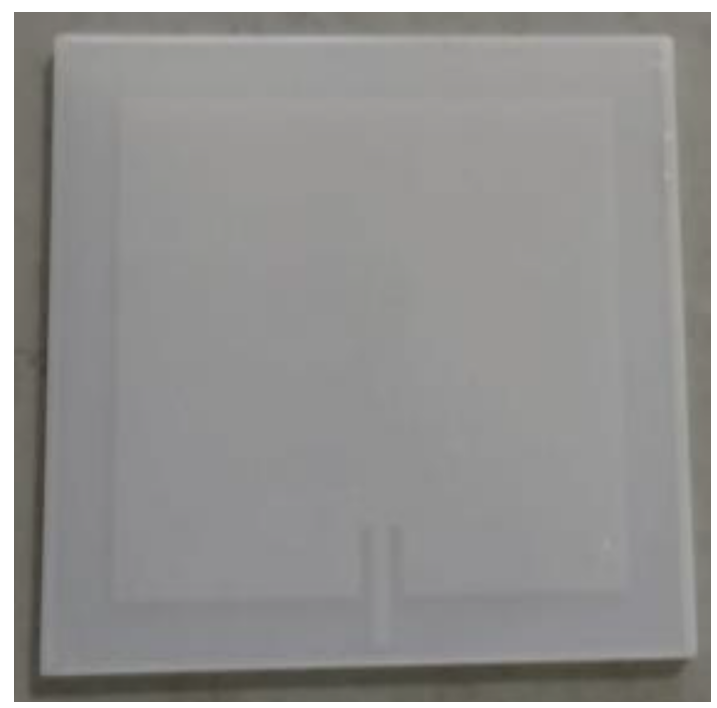

Fig. 3. Substrates mould.

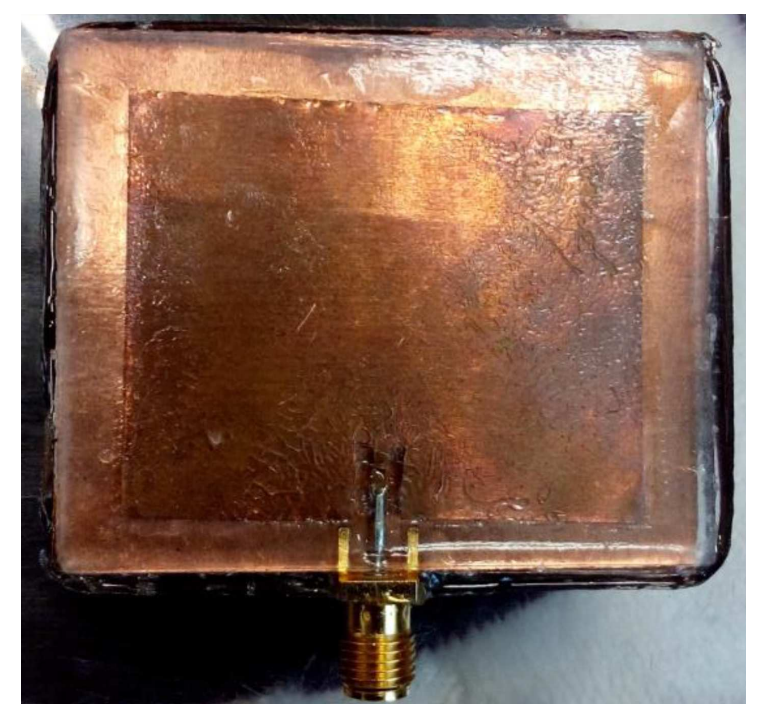

Fig. 4. Fabricated PDMS antenna .

\section{Result and discussion}

Figure 5 shows the measurement of relative permittivity of PDMS. At $2.4 \mathrm{GHz}$, the measured relative permittivity is 2.7 which is similar to the simulated value used in the design.

In Fig. 6, the graph shows the lossy tangent $\tan \delta$ of PDMS at resonant frequency $(2.4 \mathrm{GHz})$ of the proposed antenna, which is 0.01962 .

The return loss of the antenna is shown in Fig. 7. It can be seen that the antenna is having a return loss at $2.45 \mathrm{GHz}$ (ISM band) for simulated but the measured value is shifted to $2.6 \mathrm{GHz}$. This shift is expected but will be improved when the antenna is placed on the body (i.e arm) as this antenna is for wearable and body centric wireless communication.

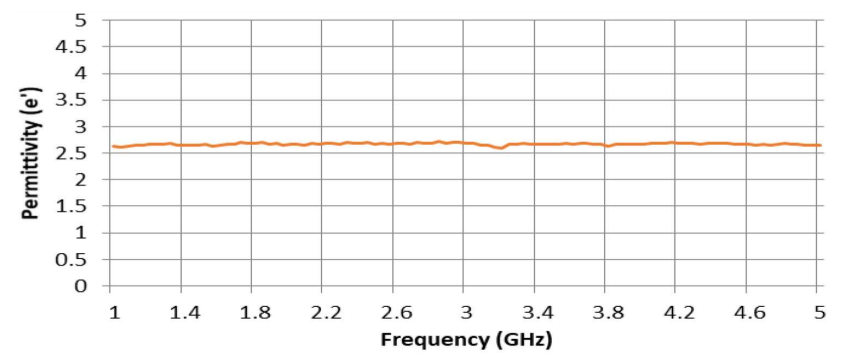

Fig. 5. Measured relative permittivity of PDMS.

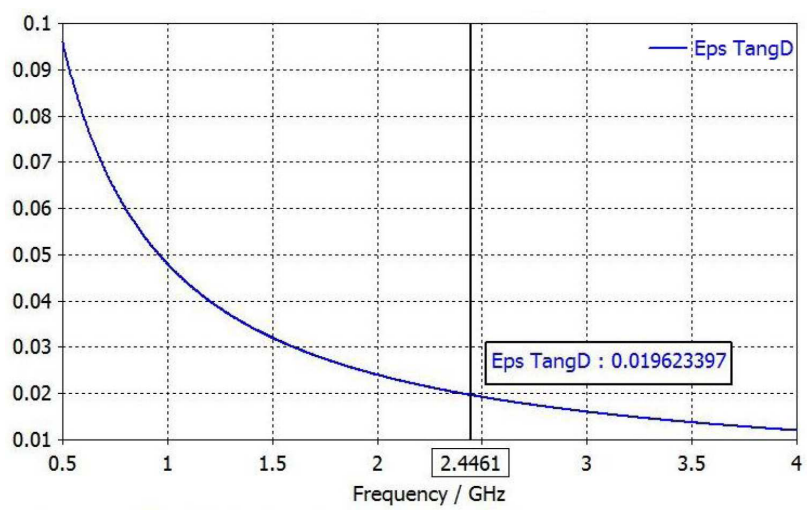

Fig. 6. Lossy tangent $(\tan \delta)$ value for the proposed antenna.

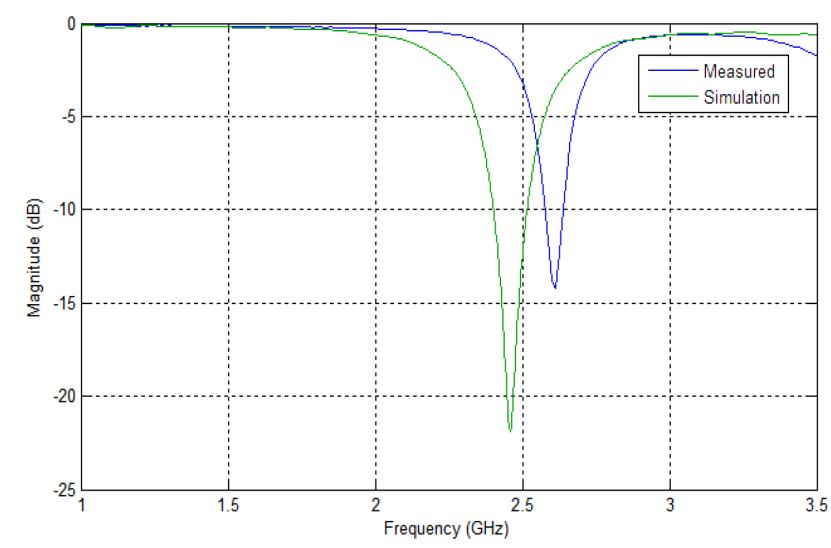

Fig. 7. Return loss - comparison of $S$-parameter between measured and simulation.

The farfield pattern of the designed antenna is shown in Fig. 8, polar view of the radiation pattern is omnidirectional because the main lobe is approximately symmetrical with the 2 minor lobe in direction of $-90^{\circ}$ and $30^{\circ}$. The energy radiated at the outermost of the antenna, having an omnidirectional pattern which is normal to the patch and is compatible for BCWCs and wearable antenna application. Main lobe is where the greatest field strength exhibits and containing the maximum power was directed on $30^{\circ}$ angle with the magnitude of $18.9 \mathrm{dBi}$. 


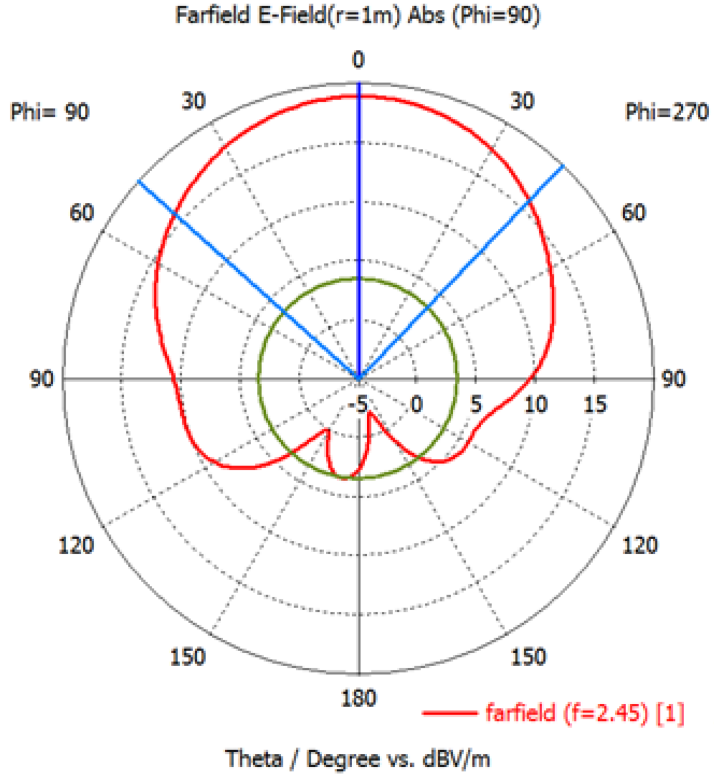

Fig. 8. Radiation pattern: frequency $=2.45 \mathrm{GHz}$, main lobe magnitude $=18.9 \mathrm{~dB} \mathrm{~V} / \mathrm{m}$, main lobe direction $=0.0^{\circ}$, angular width $(3 \mathrm{~dB})=91.7^{\circ}$, side lobe level $=-15 \mathrm{~dB}$.

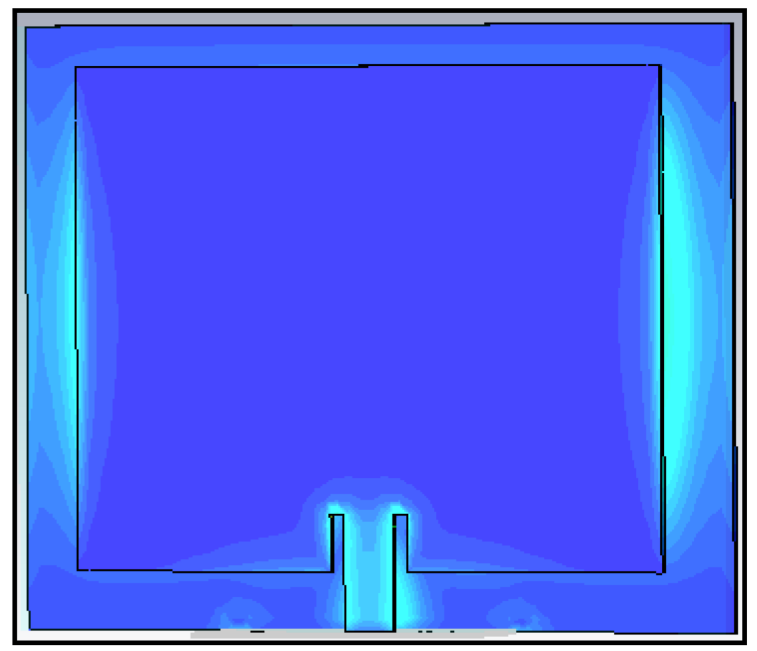

Fig. 9. Surface current with inset feed.

Bandwidth of the designed antenna was obtained through the simulation in the $S$-parameter section, the bandwidth is found to be $114.16 \mathrm{MHz}$ and good enough for a narrowband antenna application as the industrial, scientific and medical (ISM) band worldwide frequency allocation bandwidth is $2.4 \mathrm{GHz}-2.5 \mathrm{GHz}$ with $2.45 \mathrm{GHz}$ as it center frequency. Figure 9 shows the surface current for the designed antenna.

\section{Conclusions}

A simple microstrip patch antenna with obligatory specification operating frequency $2.45 \mathrm{GHz}$ is obtained, hence fulfill the condition design for industrial, scientific and medical (ISM) band. A stretchable and flexible antenna that incorporating $\mathrm{Cu}$ as radiating element and PDMS as substrate is reported in this paper. The proposed material has shown a good performance in flexibility and conductivity. PDMS has given an impact through the flexibility substrate of the proposed antenna and thermally stable.

\section{Acknowledgments}

The authors acknowledge the financial support from Universiti Teknologi MARA and Ministry of Higher Education Malaysia under Grant No: 600-RMI/FRGS 5/3 (14/2015) entitled "Wearable Flexible Polymer Based Antenna Design Incorporates Highly Conductive Radiating Element for Body Centric Wireless Communication".

\section{References}

[1] J. Trajkovikj, J.F. Zurcher, A.K. Skrivervik, IEEE Antennas Propag. Mag. 55, 287(2013).

[2] C.P. Lin, C.H. Chang, Y.T. Cheng, C.F. Jou, IEEE Antennas and Wireless Propagation Letters 10, 1108 (2011).

[3] J. Trajkovikj, J.F. Zurcher, A.K. Skrivervik, in: Proceedings of Loughborough Antennas Propagation Conference (LAPC), Loughborough 2012, p. 1.

[4] A.S. Za, S.N. Ibrahim, N.F.A. Malek, A.M. Ramly, in: Proceedings of IEEE Regional Symposium on Micro and Nanoelectronics (RSM), Batu Ferringhi 2017, p. 260.

[5] A.S.M. Alqadami, M.F. Jamlos, in: Proceedings of IEEE Asia-Pacific Conference on Applied Electromagnetics (APACE), Johor Bahru 2014, p. 27.

[6] R.B.V.B. Simorangkir, Y. Yang, L. Matekovits, K. Esselle, IEEE Antennas and Wireless Propagation Letters 16, 677 (2017).

[7] L.C. Paul, S. Hosain, S. Sarker, M.H. Prio, M. Morshed, A.K. Sarkar, American Journal of Networks and Communications 4, 54 (2015).

[8] T. Rai, P. Dantes, B. Bahreyni, W.S. Kim, IEEE Electron Device Lett. 34, 544 (2013).

[9] N. Komoda, M. Nogi, K. Suganuma, H. Koga, K. Otsuka, in: Proceedings of 2012 12th IEEE International Conference on Nanotechnology (IEEE-NANO), Birmingham 2012, p. 1.

[10] Q. Liu, K. L. Ford, R. Langley, A. Robinson, S. Lacour, in: Proceedings of 6th European Conference on Antennas and Propagation (EUCAP), Prague 2012, p. 168.

[11] H.A. Rahman S.K.A. Rahim, in: Proceedings of IEEE MTT-S 2015 International Microwave Workshop Series on RF and Wireless Technologies for Biomedical and Healthcare Applications (IMWS-BIO), Taipei 2015, p. 193.

[12] J. Trajkovikj, Z. Jean-Francois, A.K. Skrivervik, in: GeMiC 2014; German Microwave Conference, Aachen, Germany 2014, p. 1. 